Amsterdam Expeditions to the West Indian Islands, Report 40*

\title{
HESPEROCYCLOPS IMPROVISUS N. G., N. SP., EIN NEUER CYCLOPOIDE (CRUSTACEA, COPEPODA) VON DEN WESTINDISCHEN INSELN
}

\author{
von
}

\section{HANS-VOLKMAR HERBST}

\author{
Distelweg 1, 4150 Krefeld 29 - Hüls, Bundesrepublik Deutschland
}

\begin{abstract}
SUMMARY
In certain wells on the islands Antigua and Guadeloupe (West Indies), females and males of a new species of Cyclopidae were found, apparently belonging to an undescribed genus, for which the name Hesperocyclops improvisus n. g., n. sp., is proposed. The systematic position of this taxon is near Apocyclops Lindberg, 1942, but there are some morphological distinctions, e.g. in the shape of the genital somite and of the anal operculum in both sexes, in the different reduction of the 4th endopodite in females and males, and in the shape of P5. These developments may be considered an evolutionary step in the direction of the morphology of Haplocyclops Kiefer, 1952 (according to Dussart, 1982, a subgenus of Bryocyclops Kiefer, 1927).
\end{abstract}

\section{RÉSUMÉ}

Des exemplaires des deux sexes d'une nouvelle espèce de Cyclopidae, appartenant vraisemblablement à un genre nouveau, ont été capturés dans certains puits des îles Antigua et Guadeloupe (Indes occidentales). On propose pour ce taxon le nom Hesperocyclops improvisus n. g., n. sp.; celui-ci est systématiquement voisin de Apocyclops Lindberg, 1942, dont il se distingue néanmoins par des détails morphologiques: aspect du somite génitale et de l'opercule anal dans les deux sexes, réduction différente du $4 \mathrm{e}$ endopodite dans les femelles et dans les mâles, aspect du P5. A tous ces

* Report 39 is published in the same issue of this journal. The fieldwork during which the material, described in the present paper, was collected, has been financially supported by the Beijerinck-Popping Fonds (Amsterdam), the Treub Maatschappij (Utrecht), and the Netherlands Foundation for the Advancement of Tropical Research (WOTRO, The Hague). points de vue, on peut considérer le taxon nouveau comme représentant une étape évolutionnaire dans la direction de Haplocyclops Kiefer, 1952 (qui serait, d'après Dussart, 1982, un sous-genre de Bryocyclops Kiefer, 1927).

Hesperocyclops improvisus n. g., n. sp. (Abb. 1-20)

Material: - Holotypus: 1 \% . Amsterdamer Expeditionen zu den Westindischen Inseln, Sta. 78-4, Guadeloupe: Préf. Grande-Terre, östl. von Duval, Brunnen mit Eisendeckel, Pumpstation, ab 0,1 m $2 \mathrm{~m}$ Wassertiefe, in der Nähe eines kleinen Flusses; Position $16^{\circ} 24^{\prime} 18^{\prime \prime} \mathrm{N}$ $61^{\circ} 25^{\prime} 46^{\prime \prime} \mathrm{W}$; 4. April 1978; Paratypen: 2 \& ९, $10^{\circ}$ (Zoölogisch Museum Amsterdam).

Weitere Fundorte: Sta. 78-35, Antigua: Parish of St. Peter, Dorf Pares, im Maisfeld 300 m nördl. vom Wohnbereich, Brunnen, $2 \mathrm{~m}$ Durchmesser, teilweise abgedeckt, in $7 \mathrm{~m}$ Tiefe $0,2 \mathrm{~m}$ Wasserstand, Fallaub; Position $17^{\circ} 05^{\prime} 28^{\prime \prime} \mathrm{N} 61^{\circ} 45^{\prime} 10^{\prime \prime} \mathrm{W} ; 12$. April 1978; 3 ᄋ ㅇ, $60^{\circ} 0^{\prime}$, 22 Cop. (ZMA).

Sta. 78-42, Antigua: Parish of St. Paul, English Harbour Town südl. der Straße, Brunnen, ca. $3 \mathrm{~m}$ Durchmesser, kleine Oeffnung, in $2 \mathrm{~m}$ Tiefe $2 \mathrm{~m}$ Wasserstand, Salinität $720 \mathrm{mg} / \mathrm{l}$; Position $17^{\circ} 00^{\prime} 55^{\prime \prime} \mathrm{N} 61^{\circ} 45^{\prime} 43^{\prime \prime} \mathrm{W} ; 13$. April 1978; 19 ९ ९, 2 ○ $\sigma^{\circ}, 11$ Cop. (ZMA).

Beschreibung. - Das Weibchen (Abb. 1) besitzt einen relativ gestreckten Thorax und ein im Verhältnis dazu kurzes Abdomen, das reichlich die halbe Thoraxlänge erreicht $(1: 0,6)$. Das Cephalothoraxsegment ist wesentlich länger als die Thoraxsegmente 2-5 zusammengenommen, dieses Verhältnis liegt bei 1,5:1. Die absoluten Maße des Thorax beim typischen 
Weibchen sind: Cephalothoraxsegment = Länge : Breite $=241: 223 \mu \mathrm{m}$, Thoraxlänge $=$ $400 \mu \mathrm{m}$. Die Abdominalsegmente sind durch die Fixierung eingezogen (Abb. 2). Das Genitalsegment ist wesentlich breiter als lang und in seinem proximalen Teil ventrad vorgewölbt (Abb. 3). Die drei folgenden Abdominalsegmente sind kurz und breit, das 3. etwas kürzer als das Analsegment. Maße der Abdominalsegmente sind:

$\begin{array}{lccc} & \text { Länge } & \text { Breite } & \\ \text { Genitalsegment } & 99 & 138 & \mu \mathrm{m} \\ \text { 2. Abdominalsegment } & 37 & 73 & \mu \mathrm{m} \\ \text { 3. Abdominalsegment } & 30 & 66 & \mu \mathrm{m} \\ \text { Analsegment } & 33 & 61 & \mu \mathrm{m}\end{array}$

Das Receptaculum seminis (Abb. 2) nimmt bei starker Füllung den größten Teil der Ventralfläche des Genitalsegments ein. Sein proximaler Teil ist breit mit konvexem Vorderrand, der distale Abschnitt ist breit dreieckig gerundet. Die Hinterränder der Abdominalsegmente besitzen eine grob gezähnte Hyalinmembran, lediglich das Analsegment zeigt eine enge Zähnelung. Das Analoperculum (Abb. 4, 5) ist nach hinten verlängert und schließt mit einem abgestutzten, seitlich gerundeten, glatten $\mathrm{Di}$ stalrand ab.

Die Furkaläste (Abb. 4) sind kurz, zwischen 2,5-3 mal so lang wie breit. Die Lateralrandborste inseriert hinter der Mitte des Außenrandes. Von den Endborsten ist die glatte, mediale knapp halb so lang wie die laterale; letztere ist locker gefiedert und kürzer als der Furkalast. Die beiden mittleren Endborsten sind lang, befiedert, die äußere von ihnen ist über viermal, die innere über sechsmal so lang wie die laterale Apikalborste. Die unbefiederte Dorsalborste erreicht die Länge der lateralen Apikalborste, die Lateralrandborste ist halb so lang wie letztere.

Maße der Furka und ihrer Anhänge sind: Länge : Breite $=(27+16): 17 \mu \mathrm{m}=$ $(1,59+0,94): 1 .^{* *}$ Endborsten von außen nach innen $=37: 156: 236: 16 \mu \mathrm{m}=1: 4,22: 6,38$

** Das + Zeichen in der Längenangabe der Furka gibt die Insertion der Lateralrandborste an.
: 0,43 auf die laterale $=1$ bezogen. Dorsalborste $=37 \mu \mathrm{m}=1,0: 1$ und Lateralrandborste $=19 \mu \mathrm{m}=0,51: 1$ auf die laterale Endborste bezogen.

Die 1. Antenne (Abb. 6) setzt sich aus elf Gliedern zusammen, sie erreicht fast die Länge des Cephalothoraxsegments. Ihre Längenmaßc, an der Basis beginnend, sind: $49: 14: 23$ $: 12: 8: 17: 28: 23: 16: 22: 22 \mu \mathrm{m}=234 \mu \mathrm{m}$. Bau und Bewehrung der viergliedrigen 2. Antenne sind aus der Abb. 7 zu erkennen. Der rudimentäre Mandibulartaster (Abb. 8) trägt zwei lange und eine feine, kurze Borste. Das 2. Basalglied der 1. Maxille (Abb. 10) besitzt außer den drei ventrad gerichteten dorn- bzw. borstenartigen Anhängen vier rostrad gestellte Borsten, von denen zwei auf einem Vorsprung stehen. Die 2. Maxille (Abb. 9) und der Maxilliped (Abb. 11) weichen nicht bemerkenswert von dem in der Familie üblichen Bau ab.

Die Aeste der Schwimmbeine bestehen aus zwei Gliedern, lediglich der Endopodit 4 ist eingliedrig. Die Dornformel der Außenastendglieder ist 3443 , jedes dieser Glieder trägt außerdem 5 Borsten. Das 2. Basalglied des P1 (Abb. 12) ist an seiner medialen Ecke deutlich abgerundet vorgewölbt, lateral daneben inseriert ein schlanker, gefiederter Dorn. Der gekrümmte Enddorn des Endopoditen 1 ist außerordentlich stark. Die Lateralrandecke des 1. Endopoditengliedes und die Dornen an der Basis der Lateralrandborste und der Endklaue des 2. Endopoditengliedes sind als kräftige, etwas gekrümmte Kegel ausgebildet. Die Verbindungsplatte beider Schwimmbeine des P1 ist an beiden Seiten bucklig vorgewölbt. Der P2 und P3 sind gleich gegliedert und bewehrt, die Endglieder des P2 erscheinen lediglich etwas kürzer und breiter als beim P3. Bei beiden Schwimmbeinen ist die innere Ecke des 2. Basale spitz ausgezogen und die Vorwölbungen an der Verbindungsplatte sind schwächer ausgeprägt (vgl. Abb. 13). Die Form der Verbindungsplatte und des 2. Basale des P4 (Abb. 14) gleichen denen der Schwimmbeine 2 und 3. Bemerkenswert ist hier die Ausbildung des Endopoditen, der eingliedrig und auffallend breit gebaut ist. Sein Medialrand ist mit drei kurzen, befiederten 


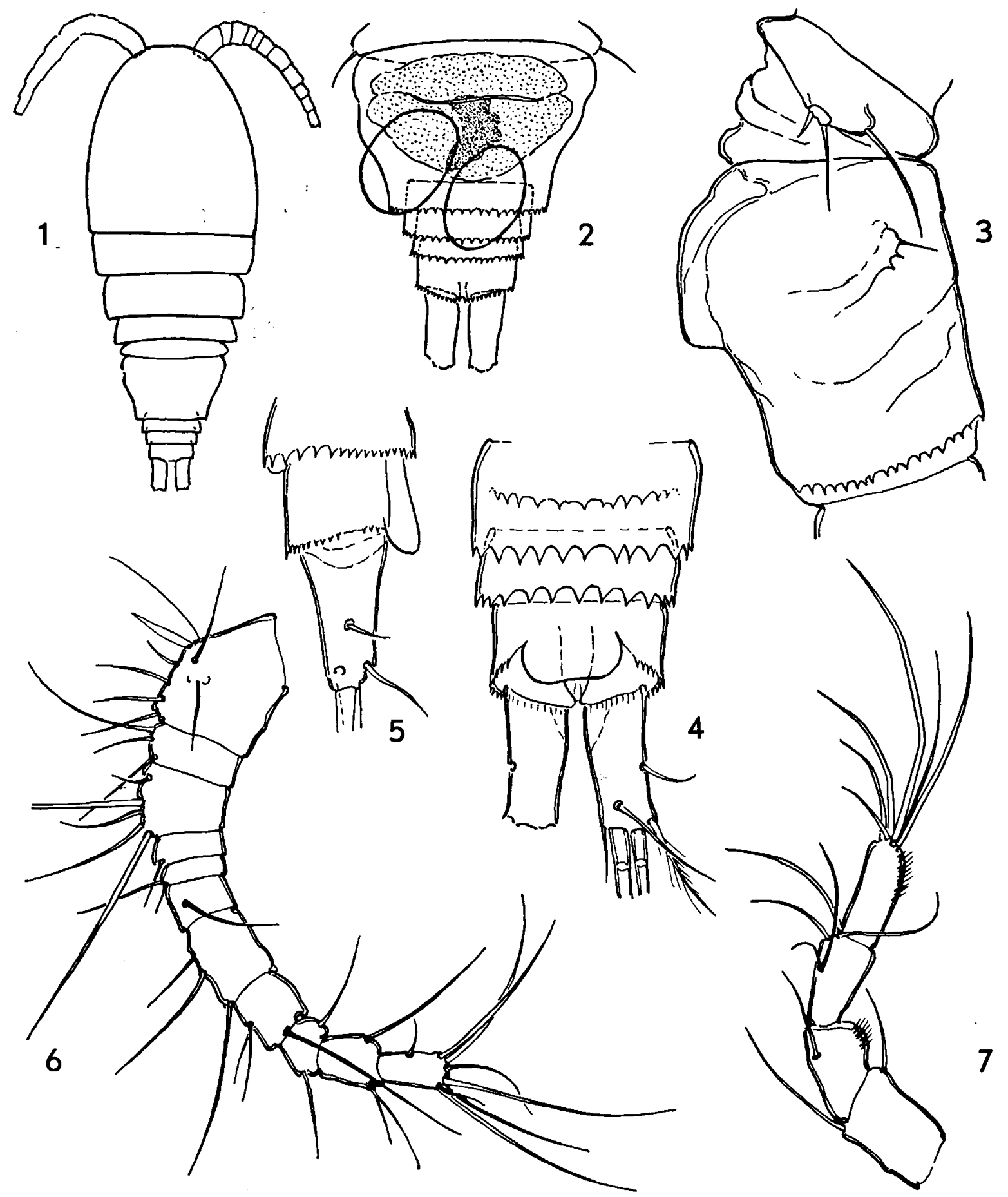

Abb. 1-7. Hesperocyclops improvisus n. g., n. sp., O Holotypus: 1, Habitus (Maßstab A); 2, Abdomen mit Receptaculum seminis und Spermatophoren (B); 3, fünftes Thoraxsegment und Genitalsegment, lateral (C); 4, Analsegment und Furka (C); 5, Analsegment und Furka, lateral (C); 6, erste Antenne (C); 7, zweite antenne (C). 
Borsten bewehrt, von denen die mittlere die geringste Länge aufweist. Am Ende stehen eine längere Borste und ein Dorn. Der Lateralrand trägt eine distal inserierende Borste und ein kurzes Dörnchen, das die ehemalige Gliedgrenze markiert. Maße des Endopoditen 4 sind: Länge : Breite $=51: 33 \mu \mathrm{m}=1,55: 1$; Borsten des Medialrandes proximal beginnend $=$ $20: 14: 21 \mu \mathrm{m}$; Borste des Apikalrandes $=35$ $\mu \mathrm{m}$, der daneben inserierende Dorn $=19 \mu \mathrm{m}$; Borste des Lateralrandes $=35 \mu \mathrm{m}$, das proximal stehende Dörnchen ist $4 \mu \mathrm{m}$ lang. Vom Grundglied des P5 (Abb. 15) ist der caudale und laterale Rand noch frei und gut zu erkennen. An der Lateralecke inseriert eine $35 \mu \mathrm{m}$ lange Borste. Das kurze Endglied ist $3 \mu \mathrm{m}$ lang und $7 \mu \mathrm{m}$ breit, es trägt zwei Anhänge, einen schlanken, medialen, $14 \mu \mathrm{m}$ langen Dorn und eine an der Basis gleich dicke, lateral stehende, $28 \mu \mathrm{m}$ lange Borste.
Zur Kennzeichnung der Variabilität der Weibchen der neuen Art werden in Tabelle I Maße der Furka und des Endopoditen 4 mitgeteilt.

Das Männchen ähnelt dem Weibchen im Habitus insofern, als auch hier das Abdomen relativ kurz und das Genitalsegment außerordentlich breit ist (Abb. 16). Das Cephalothoraxsegment nimmt mit einer Länge von $202 \mu \mathrm{m}$ etwa $60 \%$ des Thorax ein, der $328 \mu \mathrm{m}$ lang und $183 \mu \mathrm{m}$ breit ist. Die Längen-Breiten-Verhältnisse der Abdominalsegmente sind:

$\begin{array}{lccc} & \text { Länge } & \text { Breite } & \\ \text { Genitalsegment } & 69 & 137 & \mu \mathrm{m} \\ \text { 2. Abdominalsegment } & 34 & 74 & \mu \mathrm{m} \\ \text { 3. Abdominalsegment } & 27 & 57 & \mu \mathrm{m} \\ \text { 4. Abdominalsegment } & 25 & 57 & \mu \mathrm{m} \\ \text { Analsegment } & 27 & 51 & \mu \mathrm{m}\end{array}$

Das Genitalsegment ist also doppelt so breit wie lang, seine Genitalklappenbewehrung

TABELLE I

Verhältnismaße verschiedener Weibchen von Hesperocyclops improvisus n. g., n. sp.

Furka:

\begin{tabular}{lcccc}
\hline Länge : Breite & $\begin{array}{l}\text { Endborsten von außen } \\
\text { nach innen }\end{array}$ & $\begin{array}{l}\text { Dorsal- } \\
\text { borste }\end{array}$ & $\begin{array}{l}\text { Lateral- } \\
\text { randborste }\end{array}$ & Herkunft \\
\hline$(28+17): 16 \mu \mathrm{m}$ & $35: 156: 222: 16 \mu \mathrm{m}$ & $35 \mu \mathrm{m}$ & $19 \mu \mathrm{m}$ & Guadeloupe \\
$=(1,75+1,06): 1$ & $1: 4,46: 6,34: 0,46$ & 1,0 & 0,54 & \\
$(30+20): 19 \mu \mathrm{m}$ & $40: 180: 268: 23 \mu \mathrm{m}$ & $51 \mu \mathrm{m}$ & $23 \mu \mathrm{m}$ & Antigua \\
$=(1,58+1,05): 1$ & $1: 4,50: 6,70: 0,58$ & 1,28 & 0,58 & \\
$(27+19): 17 \mu \mathrm{m}$ & $44: 176: 273: 19 \mu \mathrm{m}$ & $40 \mu \mathrm{m}$ & $21 \mu \mathrm{m}$ & Antigua \\
$=(1,59+1,12): 1$ & $1: 4,00: 6,21: 0,43$ & 0,91 & 0,48 & \\
& & & &
\end{tabular}

Endopodit 4 (Verhältnisse der Anhänge auf den Apikaldorn bezogen):

\begin{tabular}{lllll}
\hline Länge : Breite & $\begin{array}{l}\text { Borsten des } \\
\text { Medialrandes }\end{array}$ & $\begin{array}{l}\text { Distal- } \\
\text { anhänge }\end{array}$ & $\begin{array}{l}\text { Anhänge des Herkunft } \\
\text { Lateralrandes }\end{array}$ \\
\hline $55: 31 \mu \mathrm{m}$ & $21: 16: 24 \mu \mathrm{m}$ & $40: 22 \mu \mathrm{m}$ & $40:-\mu \mathrm{m}$ Antigua \\
$=1,77: 1$ & $0,96: 0,73: 1,09$ & $1,82: 1$ & $1,82:-$ & \\
$54: 31 \mu \mathrm{m}$ & $28: 23: 28 \mu \mathrm{m}$ & $47: 23 \mu \mathrm{m}$ & $37: 3 \mu \mathrm{m} \quad$ Antigua \\
$=1,74: 1$ & $1,22: 1,00: 1,22$ & $2,04: 1$ & $1,61: 0,13$ & \\
\hline
\end{tabular}




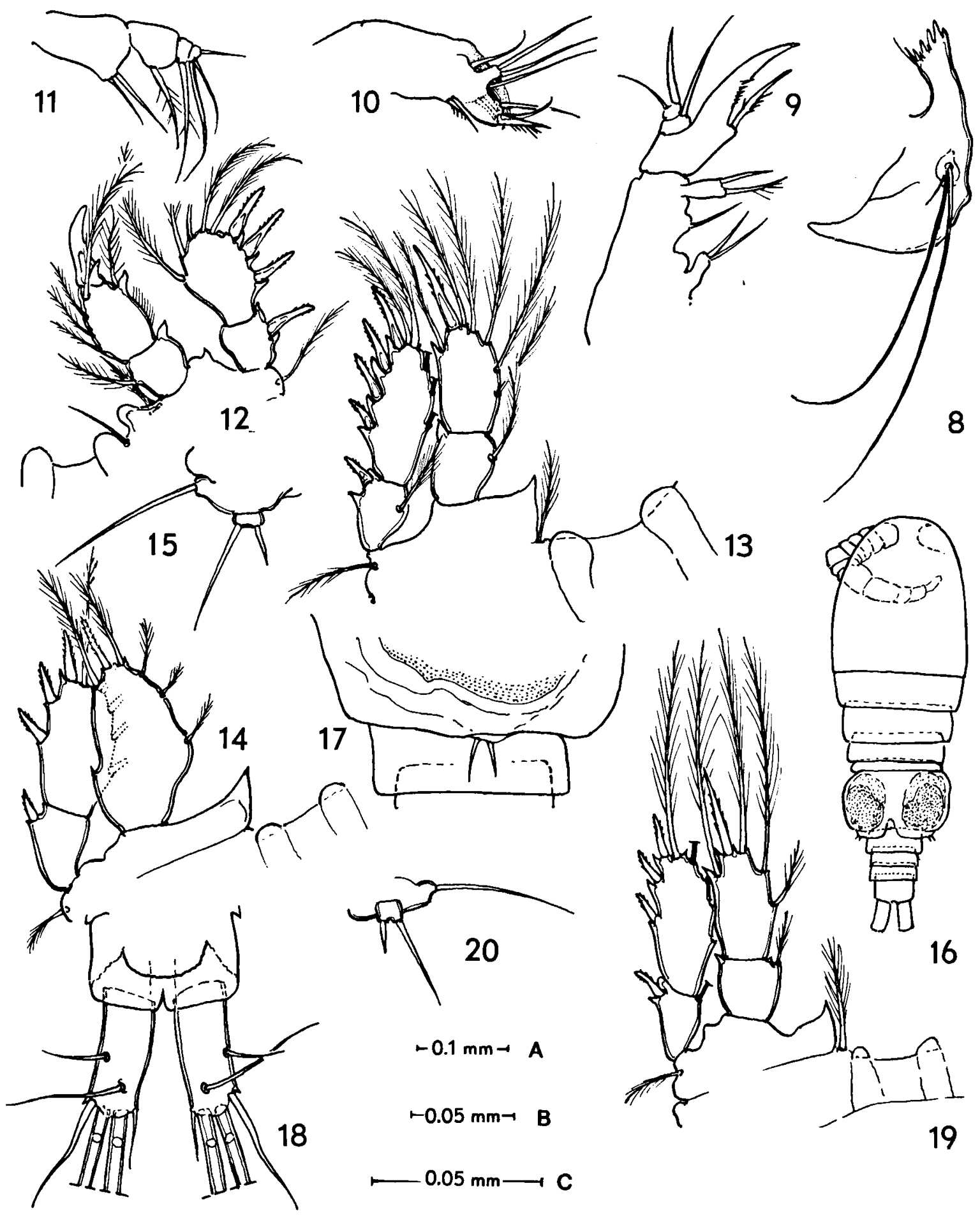

Abb. 8-20. Hesperocyclops improvisus n. g., n. sp. (8-15, ᄋ Holotypus; 16-20, ơ Paratypus): 8, Mandibel (Maßstab C); 9, zweite Maxille (C); 10, erste Maxille (C); 11, Maxilliped (C); 12, P1 (C); 13, P3 (C); 14, P4 (C); 15, P5 (C); $16, \mathrm{Habitus}$ (A); 17, Genitalsegment, Hinterrand, lateral (C); 18, Analsegment und Furka (C); 19, P4 (C); 20, P5 (C). 
(Abb. 17) besteht aus zwei fast gleichlangen und gleichstarken Anhängen, die glatt sind. Das ventrad stehende Dörnchen ist $12 \mu \mathrm{m}$, das dorsad stehende ist $14 \mu \mathrm{m}$ lang. Auch beim Männchen ist das Analoperkulum stärker entwickelt, es besitzt einen etwa halbkreisförmigen, leicht gewellten Rand (Abb. 18). Die Furka (Abb. 18) gleicht in Bau und Bewehrung der des Weibchens. Ihre Maße sind: Länge : Breite $=(26+19): 17 \mu \mathrm{m}=(1,53+1,12): 1$. Endborsten von außen nach innen $=44: 177: 257$ $: 20 \mu \mathrm{m}=1: 4,02: 5,84: 0,45$ auf die laterale $=1$ bezogen . Die Dorsalborste ist $44 \mu \mathrm{m}=1,0$ : 1 und die Lateralrandborste ist $23 \mu \mathrm{m}=0,52$ : 1 im Verhältnis zur lateralen Apikalborste lang.

Die 13-gliedrige 1. Antenne ist zum üblichen Greiforgan umgebildet. An der 2. Antenne und den Mundgliedmaßen sind keine spezifischen Unterschiede zu den gleichen Gliedmaßen des Weibchens zu erkennen.

Alle Schwimmbeine haben zweigliedrige Aeste, die Dornformel ist wie beim Weibchen 344 3, ebenso trägt jedes Exopoditenendglied fünf Borsten. Die gefiederte Borste an der Medialrandecke des proximalen Gliedes vom zweigliedrigen Endopodit 4 (Abb. 19) ist stark verkürzt. Das gleiche trifft für die proximale der am Medialrand des Endgliedes inserierenden Borsten zu. Die übrigen drei Borsten des Endgliedes distale am Medialrand, medial stehende Apikalborste, distal inserierende am Lateralrand - sind etwa gleich lang und befiedert. Der schlanke Enddorn des 4. Endopoditen ist $29 \mu \mathrm{m}$ lang. Das Endglied ist $34 \mu \mathrm{m}$ lang und $20 \mu \mathrm{m}$ breit = 1,7:1. Der P5 (Abb. 20) ähnelt in seinem Bau dem des Weibchens weitgehend, lediglich das Endglied ist weniger breit. Die Borste des Grundgliedes ist $37 \mu \mathrm{m}$ lang; am Endglied wurden die folgenden Maße festgestellt: Länge : Breite $=5: 9 \mu \mathrm{m}$, medialer Dorn $=12$ $\mu \mathrm{m}$, laterale Borste $=35 \mu \mathrm{m}$.

Wie aus der Beschreibung hervorgeht, erstreckt sich der Sexualdimorphismus bei der neuen Art auf die üblichen Merkmale (Gliederzahl des Abdomens, Ausbildung der 1. Antenne, Genitalklappenbewehrung) und außerdem auf den Bau des Endopoditen 4.
Diskussion. - Die vorstehend beschriebene, neue Art zeigt große Aehnlichkeit mit der Gruppe des Genus Apocyclops, bei der die innere Vorwölbung des 2. Basalgliedes $\mathrm{P} 4$ in eine dornförmige Spitze ausgezogen ist und die Längen der lateralen Apikalborste und der Dorsalborste der Furka etwa gleich sind. Es handelt sich nach Kiefer (1967: 301) um die drei Arten A. dengizicus (Lepeschkin, 1900), A. buxtoni (Gurney, 1921) und $A$. dimorphus (Kiefer, 1934). Allerdings sind die zahlenmäßige Verringerung der Borsten am Endopodit 4 und ihre teilweise, auffallende Verkürzung, sowie die Verschmelzung der beiden Endopoditenglieder beim Weibchen Reduktionserscheinungen, die in Richtung einer evolutionären Entwicklung hindeuten, wie sie bei Arten des Subgenus $\mathrm{Ha}$ plocyclops Kiefer, 1952 im Genus Bryocyclops Kiefer, 1927 (nach Dussart, 1982) zu finden sind. Da die morphologischen Unterschiede der neuen Art gegenüber Apocyclops ihre zwanglose Einordnung in dieses oder ein anderes bekanntes Genus nicht rechtfertigen, wird für sie ein neues Genus vorgeschlagen:

\section{Hesperocyclops n. g.}

Diagnose. - Weibchen, Thorax gestreckt, Abdomen stark verkürzt, Genitalsegment sehr kurz und breit, Analoperkulum distad verlängert, mit geradem Hinterrand und abgerundeten Ecken. Furkaläste mittellang, mediale Endborste kürzer als die laterale. Erste Antenne 11gliedrig. Beide Aeste der Schwimmbeine 1-3 und Exopodit 4 zweigliedrig, Endopodit 4 eingliedrig, Dornformel der Außenastendglieder 3 443 , Basalglied 2 des P1 mit rundlich vorgewölbten Innenecken und medialem Dorn, Basalglied 2 von P2-P4 mit dornartig vorgezogener Innenecke, Innenrandborsten am Endopodit 4 stark verkürzt. Grundglied des P5 noch erkennbar, Endglied breiter als lang, mit kürzerem medialen Dorn und längerer lateraler Borste.

Männchen, Habitus ähnelt dem des Weibchens; Aeste aller Schwimmbeine zweigliedrig, Borste des proximalen Gliedes und proximale 
Borste des Endgliedes Endopodit 4 kurz. Endglied P5 nicht so breit wie beim Weibchen; Genitalklappenbewehrung besteht aus zwei Anhängen.

Apocyclops Lindberg, 1942

Einleitung. - Aufgrund der spezifischen Ausbildung des P5 hat Lindberg (1942: 141142) die Bezeichnung Apocyclops als Subgenus von $C y c l o p s$ für die Arten $C$. dengizicus Lepeschkin, 1900, C. panamensis Marsh, 1913, und $C$. royi Lindberg, 1940, vorgeschlagen. Dieser Name wurde in der Folgezeit als Gattungsname von Lindberg (1954, 1961), Kiefer (1967), Dussart (1982) u.a. beibehalten. Außer den genannten Arten gehören die folgenden Spezies und Subspezies zu Apocyclops: $A$. dengizicus elamicus (Lindberg, 1940), A. borneoensis Lindberg, 1954, A. buxtoni (Gurney, 1921), A. dimorphus (Kiefer, 1934), A. distans Kiefer, 1956, A. japonicus Ito, 1958, $A$. panamensis tannica (Davis, 1948), A. procerus (Herbst, 1955), A. spartinus (Ruber, 1966) und $A$. viduus (Kiefer, 1933).

Eine genauere Gattungsdiagnose ist meines Wissens bisher von Apocyclops nicht gegeben worden. Für den Vergleich mit Hesperocyclops n. g. erscheint sie notwendig und soll nachstehend aufgestellt werden.

Diagnose. - Der Habitus aller Arten der Gattung ist schlank cyclopoid, das Genitalsegment des Weibchens ist etwa so lang wie breit. Das Längen-Breiten-Verhältnis der Furkaläste liegt zwischen 3-8 : 1, die medialen Endborsten der Furka sind kürzer oder höchstens so lang wie die lateralen, der Längenunterschied der beiden mittleren Endborsten ist gering (1, 1-1,3
: 1). Das Analoperkulum ist nicht spezifisch ausgebildet. Die erste Antenne ist 11-gliedrig. Die Schwimmbeinäste sind zweigliedrig, das Endglied Endopodit 4 ist mit 5 Borsten und 1 Dorn bewehrt. Vom Basalglied des P5 ist lediglich die auf einer knopfförmigen Erhebung des 5. Thoraxsegments inserierende Borste erhalten, das breite Endglied trägt an seinem Distalrand medial einen Dorn und lateral eine Borste.

Das Männchen zeigt in der Gliederung des Abdomens und der Ausbildung der 1. Antenne den üblichen Sexualdimorphismus. Die Schwimmbeine sind wie beim Weibchen ausgebildet. Die Genitalklappenbewehrung besteht aus drei Anhängen.

Die wichtigsten Gattungsunterschiede liegen danach in der Gestalt des Abdomens, speziell des Genitalsegments und des Analoperkulums, in der Form und Bewehrung des Endopoditen 4, sowie der Ausbildung des P5. Für das Männchen ist außerdem die Genitalklappenbewehrung zu nennen.

\section{LITERATUR}

Lindberg, K.,1942. Cyclopides (Crustacés Copépodes) de l'Inde, XIV-XVIII. Rec. Indian Mus., 44: 139-190.

—-, 1954. Cyclopides (Crustacés Copépodes) d'îles du Pacifique Sud (Mélanésie et Micronésie) et de Borneo. Kungl. fysiogr. Sällsk. Lund Förh., 24 (18): 1-14.

- , 1961. Remarques sur le genre Metacyclops (Kiefer 1927) et description d'un Metacyclops nouveau du Portugal. Kungl. fysiogr. Sällsk. Lund Förh., 31 (14): 133-145.

KiEfer, F., 1967. Cyclopiden aus salzhaltigen Binnengewässern Australiens (Copepoda). Crustaceana, 12 (3): 292-302.

Dussart, B., 1982. Crustacés Copépodes des eaux intérieures. Faune Madagascar, 58: 1-146. 